\title{
A potential prophylactic strategy of anti-schistosomal immunization using nanotechnology in murine models
}

\section{Original Article}

\author{
Samia E Etewa' ${ }^{1}$, Abd-Allah A Al-Hoot ${ }^{2}$, Hesham M Sharaf ${ }^{2}$, Samira M Mohammad', \\ Howayda SF Moawad ${ }^{1}$, Mohamed H Sarhan ${ }^{1}$, Mahmoud A El-Shafey ${ }^{3}$, Huda H Senosy ${ }^{2}$
}

Departments of Medical Parasitology ${ }^{1}, Z^{2}$ Zology $^{2}$ and Clinical Pathology ${ }^{3}$, Faculties of Medicine and Science, Zagazig University, Zagazig 44519, Egypt

\begin{abstract}
Background: The emergence of chemotherapy resistant schistosomes necessitates finding new prophylaxis strategies based on immunization by using a modified vaccine candidate.

Objective: To assess the potential of an anti-schistosomal vaccine modified by nanotechnology.

Material and Methods: Soluble adult worm antigen preparation (SWAP) either crude or/and loaded on chitosan nanoparticles (CSNPs) were used. The anti-schistosomal impact was assessed in terms of stools egg count, worm burden and ultrastructural study of juvenile and adult schistosomes.

Results: Chitosan nanoparticles potentiated SWAP efficacy to exceed the impact of crude SWAP. The combination of SWAP loaded on CSNPs and crude SWAP showed the best immunization effects regarding adult worm number and fecundity, as well as morphological and tegumental distortion of juvenile than adult stages.

Conclusion: Modified SWAP on CSNPs gave better results in anti-schistosome immunization than crude SWAP. Immunization by combining different antigens gave the best results.
\end{abstract}

Keywords: chitosan nanoparticles, immunization, schistosomes, SWAP, ultrastructural study.

Received: 4, November, 2018, Accepted: 11, December, 2018.

Corresponding Author: Mohamed H Sarhan, Tel.: 01020260608, E-mail: drsarhan@gmail.com

Print ISSN: 1687-7942, Online ISSN: 2090-2646, Vol. 11, No. 3, December, 2018.

\section{INTRODUCTION}

Among human parasitic diseases, schistosomiasis ranks second after malaria as a socioeconomic and public health problem in tropical and subtropical areas $^{(1)}$. It affects over 200 million people, with 20 million seriously suffering from severe anemia, chronic diarrhea, internal bleeding, and organ damage ${ }^{(2)}$. An additional 779 million individuals are at risk of acquiring the infection ${ }^{(3)}$. It was estimated that $97 \%$ of the infected individuals are concentrated in Africa ${ }^{(4)}$.

Treatment of schistosomiasis relies mainly on praziquantel (PZQ) which is distributed in mass drug administration control programs. However, program outcomes have been disappointing ${ }^{(5)}$. As PZQ has much lower efficacy against the Schistosoma juvenile stages (schistosomula) ${ }^{(6)}$, it does not prevent reinfection. Therefore, continuous drug distribution at an optimal timing is necessary to maintain disease control and break the transmission cycle $^{(7)}$. This can lead to the emergence of drug-resistant parasites and makes chemotherapy an expensive and impractical alternative. Thus, vaccination would be an important approach to complement chemotherapy ${ }^{(8)}$. So, control based on vaccination linked with chemotherapy would result in long-term protection ${ }^{(9)}$.

Of note, schistosomes don't multiply in the definitive host ${ }^{(10)}$, this is a golden chance for potent vaccines to reduce worm load via induction of effective host immune lines of defense ${ }^{(8)}$, this is associated with reduced schistosomal morbidity, pathology and transmission $^{(11)}$.

Schistosoma parasites excrete and secrete several different antigens into circulation of the host; these antigens are classified according to the stage of development of the parasite into cercarial, adult worm, and egg antigens ${ }^{(12)}$. Proteomic study of the adult SWAP revealed the intracellular nature of $80 \%$ of its recognized molecules, the gastrodermis and tegument which represent the major host-parasite interfaces contribute only $3 \%$ of signature proteins. This analysis supports SWAP as a good enhancer of the humoral and cell-mediated host immune responses ${ }^{[13]}$. Abdel-Zaher et al., ${ }^{(14)}$ used a polyvalent vaccine including SWAP and they noticed severe changes in the adult worm tegument in the form of distortion, erosion, sloughing, and atrophy of tubercles. SWAP has also an effect on the fecundity of female worms presented by the reduction in the number of $S$. mansoni eggs in stools samples ${ }^{(15)}$.

The current records of anti-Schistosoma vaccine candidates proved not to be the most effective, so it is necessary to identify new antigens and to explore alternative vaccination strategies ${ }^{(16)}$ such as the use of new adjuvants to improve vaccine efficacy ${ }^{(8)}$. However, the effective adjuvants as Freund's complete adjuvant (FCA), generally cause severe inflammation with 
unacceptable side effects ${ }^{(17)}$. It is not suitable for human application $^{(18)}$.

With the introduction of nanotechnology into the medical field, the ability of nanoparticles to elicit different immune responses through their size, and movement into cells via non-classical pathways where they are processed, was substantiated. So, efforts to develop a new delivery system for vaccines were introduced $^{(19)}$. These very small particles can transport vaccine to the site of action and provide protection against its rapid degradation or clearance. They also enhance vaccine concentration in target tissues; therefore, lower doses of vaccine are required ${ }^{(20)}$.

Chitosan is an important material for nanoparticles preparation as it is biodegradable and nontoxic. It is one of the available modified natural carbohydrate watersoluble polymers, formed by partial N-deacetylation of chitin $^{(21)}$. Chitosan nanoparticles (CSNPs) do not only act as a carrier of the vaccine but also as a good adjuvant for its enhancement, as it increases the antigen size and activates the immune system ${ }^{(22)}$. Moreover, it is used as an anti-bacterial and anti-fungal product ${ }^{(23)}$.
Many studies were done to assess anti-parasitic effects of CSNPs. It was found to produce mild protection in treatment of giardiasis ${ }^{(24)}$, increase the effect of ivermectin as anti-filarial $\operatorname{drug}^{(25)}$, and increase the therapeutic effect of spiramycin against experimental acute and chronic toxoplasmosis ${ }^{(26)}$.

The present work aims to assess the efficacy of SWAP loaded on CSNPs as a novel strategy in the prophylaxis of schistosomiasis mansoni by experimental immunization of murine models.

\section{MATERIAL AND METHODS}

This case-control experimental study was conducted in the Medical Parasitology Department, Faculty of Medicine, Zagazig University, Sharkia, Egypt, and Theodor Bilharz Research Institute (TBRI) Laboratories, Imbaba, Giza, Egypt; during the period from March, 2017 to February, 2018.

Study design: Mice were classified into five groups; each group consisted of ten mice as presented in the following table:

\begin{tabular}{ccccc}
\hline \hline Control & $\mathbf{1}$ & Control healthy & $\begin{array}{l}\text { Didn't receive any antigen or infection. } \\
\text { Didn't receive any antigen, but infected subcutaneously with } S . \text { mansoni } \\
\text { cercaria. }\end{array}$ \\
\hline Immunized & $\mathbf{2}$ & Control infected & Modified SWAP on CSNPs & $\begin{array}{l}\text { Received crude SWAP and FCA, then infected subcutaneously with S. mansoni } \\
\text { cercaria. }\end{array}$ \\
& $\mathbf{5}$ & Combined antigens & $\begin{array}{l}\text { Reced modified SWAP on CSNPs, then infected subcutaneously with } S . \\
\text { Received crude SWAP + FCA and modified SWAP on CSNPs, then infected } \\
\text { subcutaneously with S. mansoni cercaria. }\end{array}$ \\
\hline \hline
\end{tabular}

All groups were equally divided into two subgroups. Subgroups $(2,3,4$, and 5$)$ were euthanized and sacrificed on day 21 post-infection (PI), while the subgroups $\left(2{ }^{\prime}, 3^{\prime}, 4^{\prime}\right.$, and $\left.5^{\prime}\right)$ were euthanized and sacrificed on day $49 \mathrm{PI}^{(27)}$. Evaluation was by assessment of the immunization effects on eggs counts in stools by Kato technique, worm burden by hepatic perfusion, and impact on structure by scanning electron microscopy (SEM) of both juvenile and adult stages of $S$. mansoni from experimentally immunized then challenged murine models.

\section{Materials}

Experimental animals: A total of 50 healthy, laboratory-bred Swiss albino male mice weighing about 20-25 gm were obtained from the animal house, TBRI, Imbaba, Giza, Egypt. During the study, they were kept in good ventilated rooms in standard cages and provided with stock commercial pellet diet and were given water ad libitum.

Crude SWAP antigen: Schistosoma crude SWAP antigen was prepared according to the method of Salih et al., ${ }^{(28)}$.
Adjuvants: FCA was obtained from Sigma Chemical Co., St Louis, Mo, USA and emulsified in phosphate-buffered saline (PBS) at a ratio of 2:1. Chitosan (deacetylation degree of $93 \%$ ) and sodium tripolyphosphate (TPP) were purchased from Sigma-Aldrich, USA. PBS and acetic acid were obtained from Sigma-Aldrich, USA.

\section{Methods}

Ionic gelation method for CSNPs preparation: CSNPs were synthesized via the ionotropic gelation of chitosan with TPP anions. TPP was used as it is nontoxic, multivalent and has the ability for gel formation via ionic interactions. The charge density of TPP and chitosan interaction is controlled under the influence of the solution $\mathrm{pH}$. Chitosan was dissolved in various concentrations of acetic aqueous solution; 1, 2 and 3 $\mathrm{mg} / \mathrm{ml}$. The chitosan concentration was 1.5 times lower than that of acetic acid in aqueous solution. The TPP solution $(1 \mathrm{mg} / \mathrm{ml})$ was prepared in double-distilled water. CSNPs were prepared by dropwise addition of $5 \mathrm{ml}$ of the chitosan solution to $2 \mathrm{ml}$ of TPP solution while on $1000 \mathrm{rpm}$ magnetic stirring for $1 \mathrm{~h}$ at room temperature. The suspension was performed under the same above-mentioned conditions. Separations of 
nanoparticles were done by centrifugation at $20000 \mathrm{~g}$ at $14^{\circ} \mathrm{C}$ for $30 \mathrm{~min}$, and then they were freeze-dried and stored at $4^{\circ} \mathrm{C}$. Their weights were measured, and they were characterized using SEM (JEOL $100 \mathrm{CX})^{(29)}$.

Modified SWAP antigen/CSNPs preparation: Chitosan solution was added to TPP solution containing SWAP at a concentration of $100 \mathrm{mg} / \mathrm{ml}$. The combined chitosan and SWAP were separated from the suspension by centrifugation $\left(20000 \mathrm{~g}\right.$ at $\left.14^{\circ} \mathrm{C}\right)$ for $30 \mathrm{~min}$. The supernatant was collected and protein content (free SWAP) in supernatant was determined by the Bradford protein assay spectrophotometric method at $595 \mathrm{~nm}$. The SWAP encapsulation efficiency (AE) and loading capacity (LC) of nanoparticles were calculated as follows:

$\% \mathrm{AE}=[(\mathrm{A}-\mathrm{B}) / \mathrm{A}] \times 100$

$\% \mathrm{LC}=[(\mathrm{A}-\mathrm{B}) / \mathrm{C}] \times 100$

Where A is the total amount of SWAP, B is the free amount of SWAP, and $C$ is the weight of nanoparticles.

Parasite (S. mansoni, Egyptian strain): Collection of cercariae was done according to Liang et al., ${ }^{(30)}$. Cercariae were obtained from infected Biomphalaria alexandrina snails purchased from TBRI following exposure to artificial light to induce cercariae shedding in the laboratory.

Estimation of cercarial densities: The average number of cercariae was determined per one $\mathrm{ml}$ of cercarial suspension, then the suspension was subsequently diluted by distilled water to the desired number of cercariae per one $\mathrm{ml}$ of suspension for animal infection according to Webbe and James ${ }^{(31)}$.

Mice infection: Each mouse was infected through the abdominal skin by a subcutaneous injection of $80 \pm 10$ pre-harvested cercariae of $S$. mansoni 3 weeks after the initial subcutaneous antigen injection $\left(1^{\text {st }}\right.$ immunization dose $)^{(32)}$

Regimen of vaccination: According to Nabih and Soliman ${ }^{(33)}$, each mouse of the tested groups was sensitized with an initial subcutaneous injection of 200 $\mu \mathrm{l}$ of the extracted SWAP antigens (either crude or loaded on CSNPs) with a total antigen concentration of $30 \mu \mathrm{g}$ protein. After two weeks from initial injection, a second injection composed of $200 \mu \mathrm{l}$ of the same antigens diluted by PBS to contain $20 \mu \mathrm{g}$ protein, was administered. The injection was composed of the antigen combined with FCA at a 1:1 ratio, to the test groups 3 and $5^{(34)}$.

Assessment parameters: The following studies were performed for infected groups.

\section{Parasitological studies}

1. Modified Kato thick smear ${ }^{(35)}$ to detect the egg count/gram of stools, which was taken from the rectum at $49^{\text {th }}$ day PI (subgroups $22^{\prime}, 3^{\prime}, 4^{\prime}$, and $5^{\prime}$ ) to test the effect of immunization on the fecundity of adult worms.

2. Porto-mesenteric perfusion for estimation of worm burden ${ }^{(36)}$. Adult worms were counted and sexed under a stereomicroscope. Morbidity was determined by the percentage of maturation of cercariae to adult worms recovered from the portal system and mesenteric veins. The percentage reduction of worm burden in all immunized and challenged subgroups was calculated according to the following equation ${ }^{(37)}: \mathrm{R} \%=[($ Mean of worm burden recovered from control group - Mean of worm burden recovered from tested group)/Mean of worm burden recovered from control group)] X 100.

Ultrastructural study by SEM $^{(38)}$ : This study was performed at Electron Microscope Unit, Faculty of Agriculture, Mansoura University, Egypt. Worms either juveniles or adults were obtained by perfusion of the hepatic portal blood vessels of immunized and challenged subgroups at $21^{\text {st }}$ and $49^{\text {th }}$ days PI, respectively. They were first washed carefully by shaking gently in distilled water to which a few drops of detergent solution were added to dislodge any attached debris. Then, they were fixed in $2.5 \%$ glutaraldehyde-phosphate buffer $(0.1 \mathrm{~mol} / \mathrm{l}, \mathrm{pH} 7.4)$ at $-4^{\circ} \mathrm{C}$ for $24 \mathrm{~h}$ and post fixed in $1 \%$ osmium tetroxide for $1 \mathrm{~h}$. They were dehydrated through graded series of ethanol and dried in a Hitachi HCP-2 (Hitachi Koki, Tokyo, Japan) critical-point dryer machine using liquid carbon dioxide as a transitional medium. After drying, they were mounted on aluminum stubs and coated with platinum and palladium in an ionsputtering apparatus (Hitachi E-102) at 10-15 $\mathrm{mA}$ for 6 min. Profiles of worms were examined using Joel JEM1200 SEM.

Statistical analysis: Data were analyzed using the Statistical Package for Social Science (SPSS) for windows version 20.0 with student $t$ test and ANOVA test to evaluate the possible differences between the study groups. $P$ value $<0.05$ was considered significant.

Ethical considerations: The study was approved by the ethical committee of the Faculty of Medicine, Zagazig University, Egypt, according to the international guiding principles for biomedical research involving animals as issued by the international organizations of medical sciences. All animals received humane care, and the study protocol complies with the guide lines of Institutional Animal Care and Use Committee (IACUC), Zagazig University.

\section{RESULTS}

Nanoparticles characterization: SWAP-loaded CSNPs had a solid and consistent structure, being rounded, regular, and had a smooth surface. Their mean size was $60.08 \pm 2.009 \mathrm{~nm}$ By SEM. 
S. mansoni immunization using nanotechnology Etewa et al.

Parasitological parameters: Results of egg count per gram stools by the Modified Kato technique in both control and tested groups (Table 1) showed significant reduction $(P<0.001)$ in the number of $S$. mansoni eggs in fecal samples 7 weeks PI in the tested subgroups $\left(3^{\prime}, 4^{\prime}\right.$, and 5 ') compared with the infected control subgroup (2') with percentage reduction of $81.4 \%, 85.2 \%$ and $91.3 \%$, respectively. While on comparing the results of the tested subgroups $\left(3^{\prime}, 4^{\prime}\right.$, and $\left.5^{\prime}\right)$ together, we found a significant reduction in egg count in subgroup (5') more than subgroup (4') and the least reduction was in subgroup (3`).

All tested groups $(3,4$, and 5$)$ showed significant reduction in the total worm burden compared with the infected control group $2(P<0.001)$. On comparing them together, the lowest total worm burden $(6.69 \pm 3.50)$ was detected in mice of group 5 (combined SWAP, then challenged by schistosomiasis), followed by group 4 (modified SWAP loaded on CSNPs, then infected) $(10.58 \pm 3.75)$ and the least reduction was in group 3 (crude SWAP+FCA, then challenged by schistosomiasis) (13.63 \pm 4.05$)$ (Table 2).

All subgroups (3, 4, and 5) showed a significant reduction in schistosomula worm burden compared with the infected control subgroup $2(P<0.001)$. On comparing these tested subgroups with each other, the

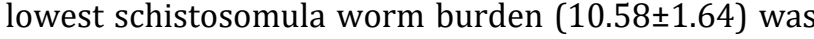
detected in mice in subgroup 5 (combined SWAP, then challenged), followed by subgroup 4 (modified SWAP loaded on CSNPs, then infected) $(14.25 \pm 1.75)$ and the least reduction was in subgroup 3 (crude SWAP+FCA, then challenged) $(17.85 \pm 1.92)$ (Table 3$)$.

All tested subgroups (3', 4', and 5') showed significant reduction in adult worm burden compared with the infected control subgroup (2') $(P<0.001)$. On comparing them together, the lowest adult worm burden $(2.8 \pm 1.1)$ was detected in mice immunized with combined SWAP, then infected (subgroup 5'), followed by modified SWAP loaded on CSNPs, then challenged (subgroup 4) $(6.9 \pm 1.1)$ and the least reduction was in crude SWAP+FCA, then infected (subgroup 3') $(9.4 \pm 1.4)$ (Table 4).

The percentage reduction (R) in mean number of mature worm burden was $85.4 \%, 64.1 \%$ and $51.0 \%$ in subgroups 5', 4', and 3', respectively. Table (5) showed significant reduction rate of couples (R 93.3\%) in subgroup 5', followed by subgroup 4 (R 79.6\%) and the least reduction in coupling was in subgroup 3` $(R$

Table 1. Modified Kato technique results (egg count per gram stools) in both control and tested groups.

\begin{tabular}{|c|c|c|c|c|c|c|}
\hline & \multirow{2}{*}{ Subgroups } & \multirow{2}{*}{ Mean \pm SD } & \multirow{2}{*}{$\mathbf{R} \%$} & \multicolumn{3}{|c|}{ Statistical analysis } \\
\hline & & & & $T$-test & $F$-test & $P$ value \\
\hline 2 & Infected control & $356.75 \pm 36.54$ & -- & -- & \multirow{4}{*}{260.01} & \multirow{4}{*}{$<0.001^{*}$} \\
\hline 3 & Crude SWAP + FCA & $66.36 \pm 7.90^{*}$ & 81.4 & 19.004 & & \\
\hline 4 & Modified SWAP loaded on CSNPs & $52.79 \pm 8.74^{*}$ & 85.2 & 19.384 & & \\
\hline 5 & Combined SWAP & $31.04 \pm 8.23^{*}$ & 91.3 & 20.798 & & \\
\hline
\end{tabular}

* Significant difference from infected control group at $P<0.001$

Table 2. Total worm burden in all studied groups (21 and 49 days post-infection).

\begin{tabular}{lcccc}
\hline \hline & Groups & Total worm burden & Statistical analysis \\
\cline { 3 - 5 } & & Mean \pm SD & Kruskal-Wallis test & P value \\
\hline \multirow{2}{*}{ Control } & $\mathbf{1}$ & - & & $<0.001^{*}$ \\
& $\mathbf{2}$ & $24.43 \pm 7.20$ & \multirow{2}{*}{32.04} & \\
\multirow{2}{*}{ Tested } & $\mathbf{3}$ & $13.63 \pm 4.05$ & & \\
& $\mathbf{4}$ & $10.58 \pm 3.75$ & & \\
& $\mathbf{5}$ & $6.69 \pm 3.50$ & & \\
\hline \hline
\end{tabular}

$\overline{\text { * Significant at } P<0.001}$

Table 3. Worm burden of $S$. mansoni schistosomula stage in the studied subgroups (2, 3, 4, and 5) (21 days post-infection)

\begin{tabular}{lcccc}
\hline \hline & \multirow{2}{*}{ Groups } & \multicolumn{2}{c}{ Schistosomula stage worm burden } & \multicolumn{2}{c}{ Statistical analysis } \\
\cline { 2 - 5 } & $\mathbf{2}$ & Mean \pm SD (R\%) & F-test & P value \\
\hline Control & $\mathbf{3}$ & $29.65 \pm 1.73$ & & $<0.001^{*}$ \\
\multirow{2}{*}{ Tested } & $\mathbf{4}$ & $17.85 \pm 1.92(39.8)$ & 62.94 & \\
& $\mathbf{5}$ & $14.25 \pm 1.75(51.9)$ & & \\
\hline * Significant at $P<0.001$ & $10.58 \pm 1.64(64.3)$ & & \\
\hline
\end{tabular}


$57.8 \%$ ). Male worms were more affected than females in the different tested subgroups, the reduction percentage of male worms was $89 \%, 71 \%, 60 \%$ in subgroups (5),
$4{ }^{\prime}$, and $\left.3^{\prime}\right)$, respectively, while it was $81.5 \%, 56.5 \%$, and $41.3 \%$ for female worms in the same studied subgroups (Table 5).

Table 4. Worm burden of $S$. mansoni adult stage in different studied subgroups (2`, 3`, 4`, and 5`) (49 days post-infection)

\begin{tabular}{|c|c|c|c|c|}
\hline \multirow{2}{*}{\multicolumn{2}{|c|}{ Groups }} & \multirow{3}{*}{$\begin{array}{c}\text { Adult stage worm burden } \\
\text { Mean } \pm \text { SD } \\
19.20 \pm 1.6 \\
\end{array}$} & \multicolumn{2}{|c|}{ Statistical analysis } \\
\hline & & & Kruskal-Wallis test & $P$ value \\
\hline Control & 2 & & \multirow{4}{*}{38.75} & \multirow{4}{*}{$<0.001^{*}$} \\
\hline \multirow{3}{*}{ Tested } & 3 & $9.4 \pm 1.4$ & & \\
\hline & 4 & $6.9 \pm 1.1$ & & \\
\hline & 5 & $2.8 \pm 1.1$ & & \\
\hline
\end{tabular}

* Significant at $P<0.001$

Table 5. Effect of the different antigenic preparations on mature worm burden of $S$. mansoni in the different studied subgroups (49 days PI) (regarding worms' sex and coupling).

\begin{tabular}{cccccc}
\hline \hline \multirow{2}{*}{ Subgroups } & \multicolumn{3}{c}{ Mean mature worm burden (\% reduction) } & Statistical analysis \\
\cline { 2 - 6 } & & Couple (R\%) & Male (R\%) & Female (R\%) & Total adult worms (R\%) \\
\hline Control & $\mathbf{2}$ & $9 \pm 1.74$ & $10.0 \pm 2.14$ & $9.20 \pm 1.20$ & $19.20 \pm 1.6$ \\
\hline \multirow{3}{*}{ Tested } & $\mathbf{3}$ & $3.80 \pm 1.50(57.8)$ & $4.0 \pm 1.05(60.0)$ & $5.4 \pm 1.64(41.3)$ & $9.4 \pm 1.4(51.0)$ \\
& $\mathbf{4}$ & $1.84 \pm 0.75(79.6)$ & $2.9 \pm 1.02(71.0)$ & $4.0 \pm 1.05(56.5)$ & $6.9 \pm 1.1(64.1)$ \\
\hline \hline
\end{tabular}

* Significant at $P<0.001$

Ultrastructure parameter: SEM of $S$. mansoni juvenile male and female worms in subgroups 2, 3, 4, and 5 (21 days post-challenged by schistosomiasis) are presented in figures (1-4). Subgroup (2) infected juvenile female showed poorly developed tegument tubercles and spines (Fig. 1). In subgroup (3), a vaccinated juvenile female (crude SWAP+FCA, then infected) showed stunted eroded tubercles on the tegument, with sessile knobs and depressions; suckers showed few blebs and swelling (Fig. 2). Subgroup (4) showed juvenile vaccinated male (modified SWAP on CSNPS, then infected) with tegumental distortion and areas of erosion; red blood cells and irregular big cells adherent to the tegument and irregular edges of gynecophoric canal (Fig. 3). Subgroup (5) showed juvenile vaccinated male with combined vaccine (SWAP + FCA + modified SWAP on CSNPs) with less developed and irregularly distributed tegumental tubercles showing loss of spines and stunted apically directed spines. Inter-tubercular ridges of the tegument were less prominent (Fig. 4).

SEM of $S$. mansoni adult worms (49 days postchallenged by schistosomiasis) are shown in figures (5-16). Normal male and female worms and in copula were obtained from subgroup (2') (Figs. 5-8). The female tegument was smoother than that of the male and covered with smoothly-developed tubercles (Fig. 5). The male tegument had large tubercles covered with spines all over the body, and the ventral sucker spines were smaller than those on the oral sucker (Fig. 6).

In subgroup (3') vaccinated with SWAP + FCA, then challenged (Figs. 9-11), there was distortion of male tegument tubercles dorsally and disruption of the borders of gynecophoric canal with protrusion of female from the canal (Fig. 9) as well as wrinkling and folding of oral and ventral suckers (Fig. 10). In addition, the male tegument showed some loss of tubercles, irregular intertubercular ridges, many irregular large cells that may be immune cells adherent to tubercles as well as red blood cells (Fig. 11).

Subgroup (4') vaccinated with modified SWAP on CSNPs, then challenged (Figs. 12-14) showed partial detachment of male tegument and loss of tubercles in some areas, distorted edges with relaxed and dilated gynecophoric canal and coiled parts of the female protruding from the canal as if preparing for separation (Fig. 12). The female tegument appeared smooth with nearly absent tubercles (Fig. 13). In other male worms, there was apparent collapse of tegumental tubercles in some areas and absence in others, tubercular spines appeared stunted and sometimes detached but the inter-tubercular ridges were mostly intact (Fig. 14).

Subgroup (5`) vaccinated with combined vaccine (SWAP+FCA+modified SWAP on CSNPs, then challenged) (Figs. 15-19) resulted in contracted shortened copulas of male and female worms with dilated gynecophoric canal and distorted edge (Fig. 15). Male worms showed some blebs in the oral sucker, edema and irregularity of the ventral sucker (Fig. 16). Tegument of female worms showed a banded appearance with loss of ridges, apparent pitting, erosion and detachment. Irregular shaped cells were noticed adherent to the tegument (Fig. 17). A single possibly separated female appeared kinked with intensive contraction and bending of the body in some regions (Fig. 18). Male tegument also showed areas of extensive erosion, stunted tubercles with nearly complete loss of spines, some blebs and edematous inter-tubercular ridges (Fig. 19). 
(1)

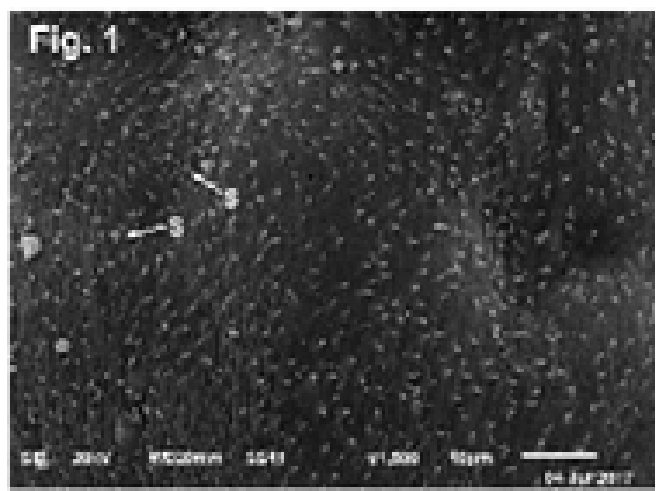

(3)

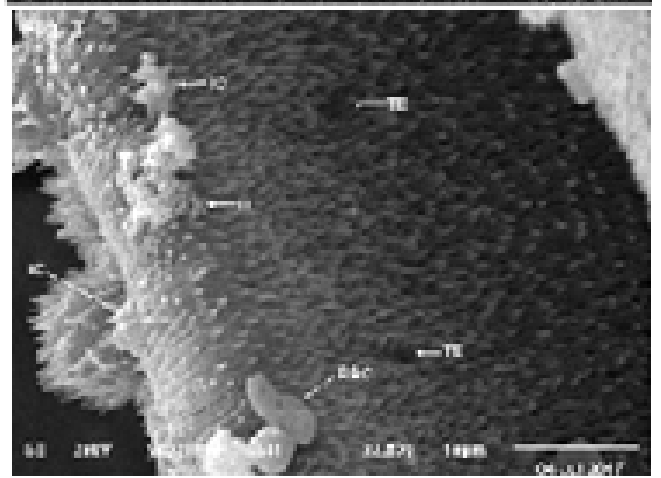

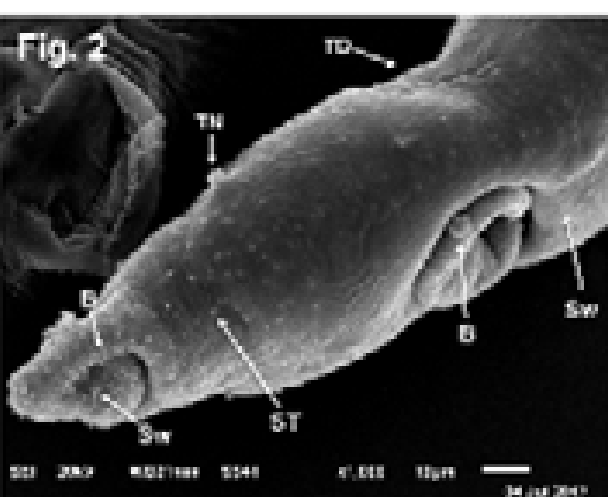

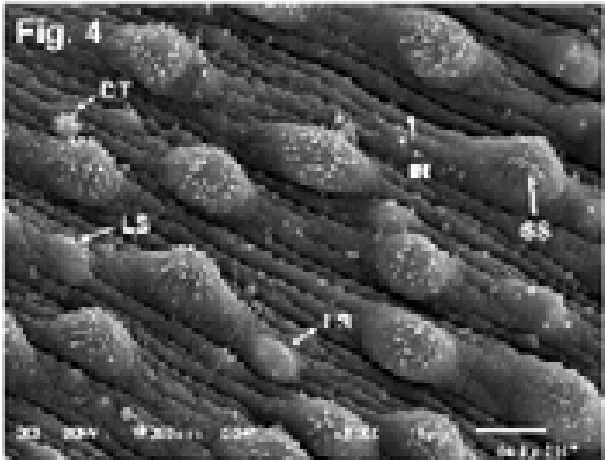

(2)

(4)

Figures 1-4: SEM of juvenile males and females

Fig. 1: Subgroup (2) (infected only): Juvenile female with not well-developed tegumental tubercles and spines (S).

Fig. 2: Subgroup (3) (crude SWAP+FCA, then infected): In juvenile female, both suckers show few blebs (B) and swelling (Sw), stunted tubercles (ST) with erosion, tegumental depression (TD). A sessile tegumental knob (TN) is noticed.

Fig. 3: Subgroup (4) (modified SWAP on CSNPs, then infected): Juvenile male showed tegumental distortion (D) with areas of erosion (TE), red blood cells (RBCs) and irregular large cells (IC) adherent to the tegument while the edges of gynecophoric canal are irregular (IE).

Fig. 4: Subgroup (5) (combined SWAP, then infected): Juvenile male showing tegumental tubercles less developed and irregularly distributed, some tubercles showing loss of spines (LS), others with stunted apically directed spines (SS). Detached part of the tegument is evident (DT) while the intertubercular ridges appear less prominent (IR).

(5)

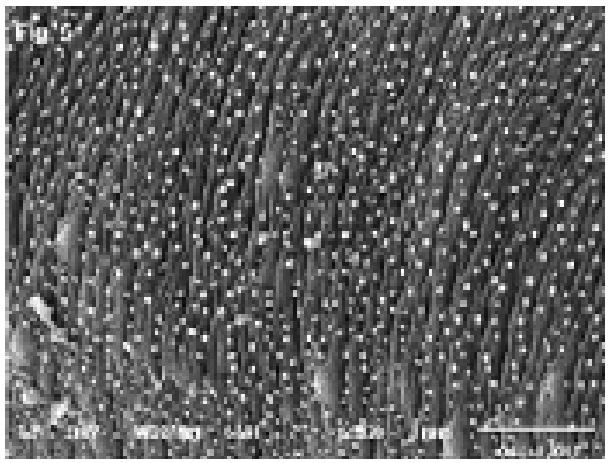

(7)

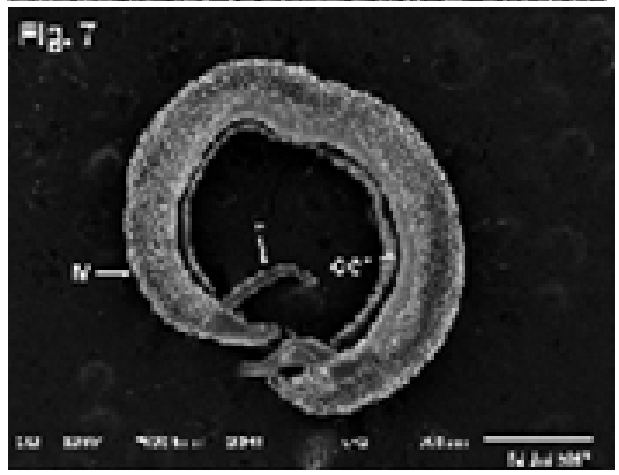

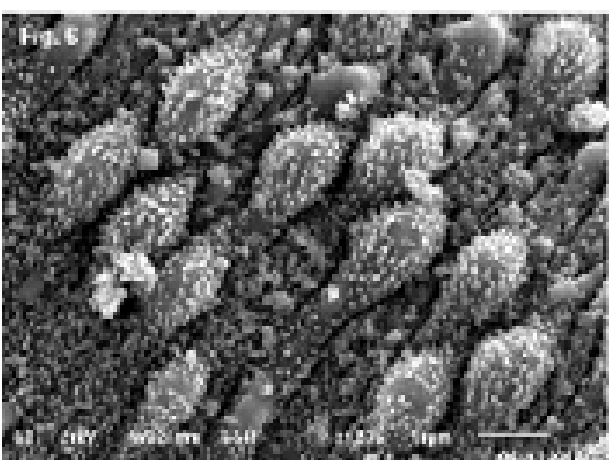

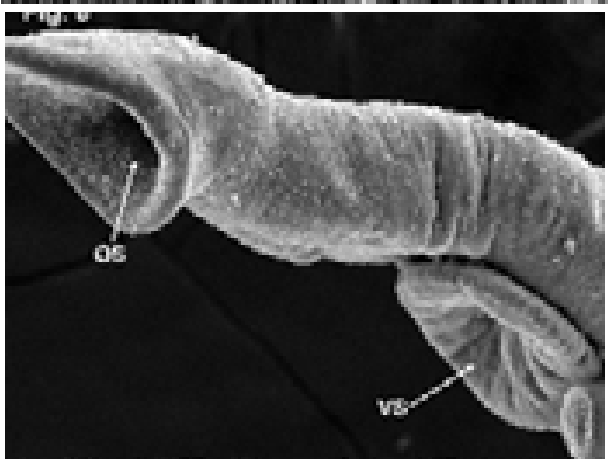

(6)

(8)

Figures 5-8: SEM of normal males and females (subgroup 2')

Fig. 5: Female tegument with clear tubercles and spines.

Fig. 6: Male tegument showing large tubercles with numerous prominent apically directed spines, and normal inter-tubercular spaces with sound ridges. Round cells are noticed on the tegument but not adherent to it.

Fig. 7: Normal configuration of mated S. mansoni worms; male (M); female (F) and gynecophoric canal (GC).

Fig. 8: Oral sucker (OS) and ventral sucker (VS) of an adult male worm. 


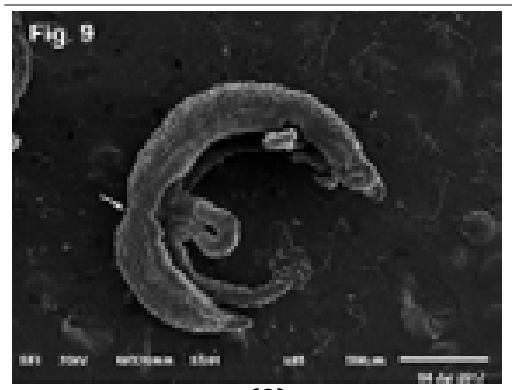

(9)

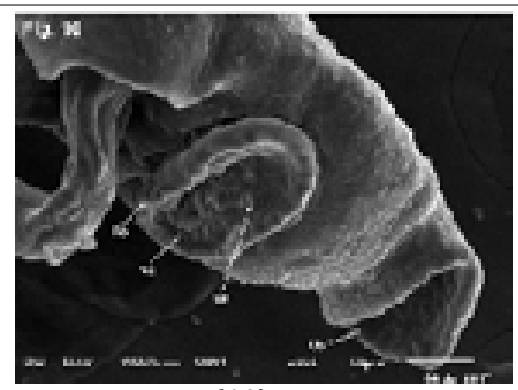

(10)

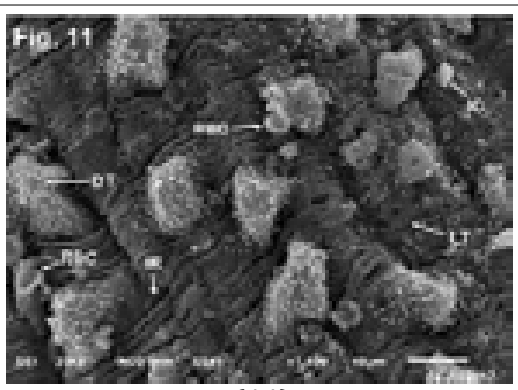

(11)

Figures 9-11: SEM of schistosomes vaccinated with SWAP + FCA, then challenged (subgroup 3')

Fig. 9: Male and female in copula showing disruption of the borders of gynecophoric canal with longer parts of the female protruded from the canal, and distortion of tegument tubercles in dorsal view of male (arrow).

Fig. 10: Male worm with changes in oral (OS) and ventral (VS) suckers with wrinkling (W) and foldings (Fo).

Fig. 11: Male tegument showing irregular distorted tubercles (DT) (arrow), some tubercles appear lost (LT), irregular intertubercular ridges (IR), many irregular large cells (IC) are present (may be immune cells) adherent to tubercles, red blood cells (RBC) are apparent.

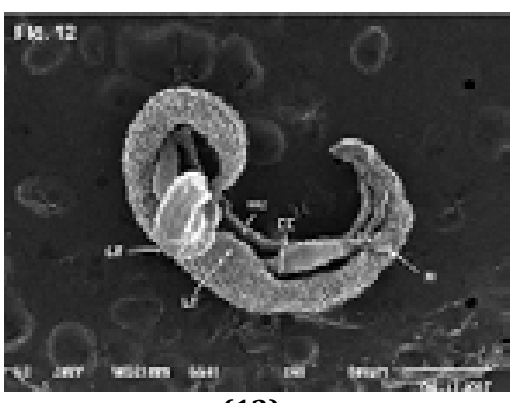

(12)

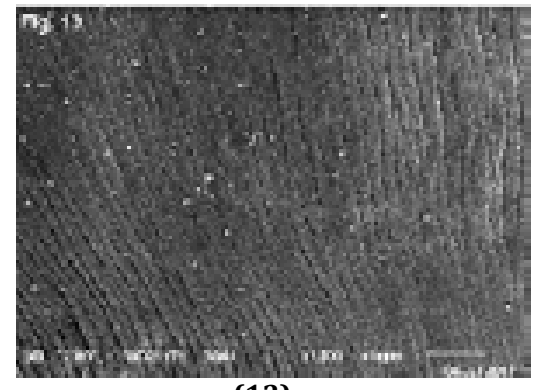

(13)

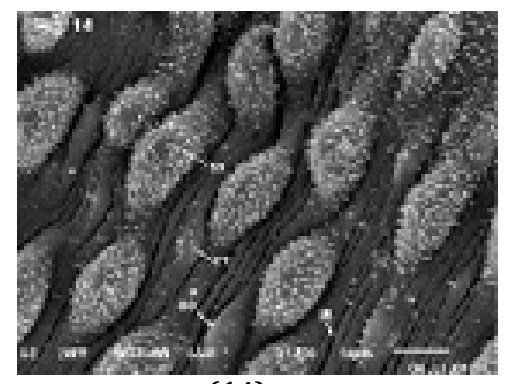

(14)

Figures 12-14: SEM of schistosomes vaccinated with modified SWAP on CSNPs, then challenged (subgroup 4')

Fig. 12: Detached part of the male tegument (DT), areas of lost tubercles (LT), distorted edges (DE) with relaxation and dilatation (expansion) of the gynecophoric canal (DC). Coiled parts of the female (CF) are protruding from the canal as if separated (or being isolated).

Fig. 13: Smooth female tegument with nearly absence of tubercles.

Fig. 14: Male tegument showing collapse of tubercles (CT) and absence of some tubercles. Tubercular spines appear stunted (SS), sometimes detached (DS) but the inter-tubercular ridges (IR) appear more or less intact.

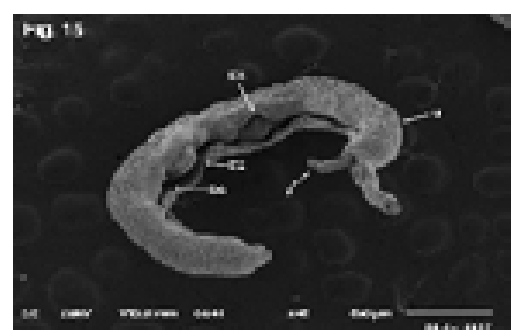

(15)

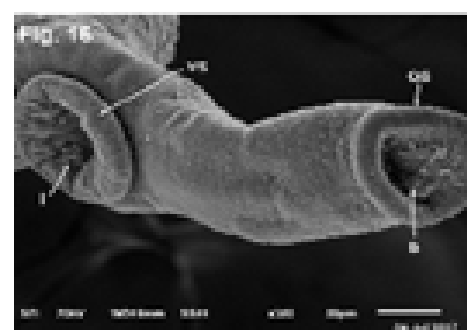

(16)

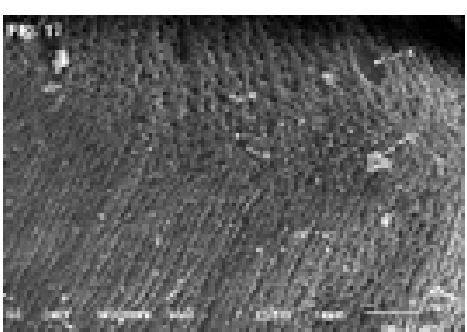

(17)

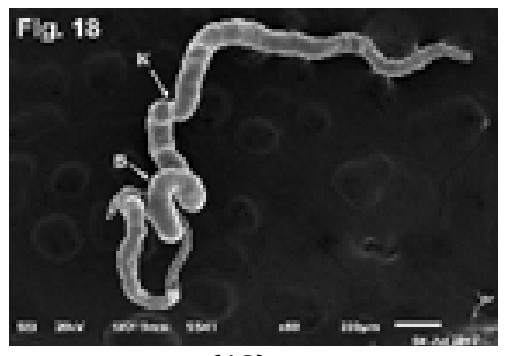

(18)

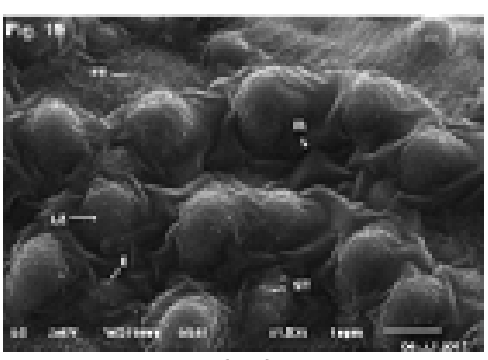

(19)

Figures 15-19: SEM of schistosomes vaccinated with SWAP+FCA+modified SWAP on CSNPs, then challenged (subgroup 5') Fig. 15: Male (M) and female (F) in copulation. They appear more contracted, curved and shortened in comparison to subgroup 4 images. The gynecophoric canal appears dilated (DC) with areas of distortion of its edges (DE).

Fig. 16: Male showing some blebs (B) of the oral sucker (OS), edema and irregularity (I) of the ventral sucker (VS).

Fig. 17: Female tegument of banded appearance, loss of ridges, apparent tegumental pitting (P), erosion and detachment (E). Irregular shaped cells (IC) appear adherent to the tegument.

Fig. 18: Whole mount of single separated female showing hypertension that is clearly apparent as kinking (K). Intensive contraction and bending (B) of the body in some regions are noticed.

Fig. 19: Male tegument showing areas of extensive erosion (EE), stunted tubercles (ST), and tubercles showing nearly complete loss of spines (LS) with some blebs (B) and edematous intertubercular ridges (IR). 


\section{DISCUSSION}

Schistosomiasis is a chronic debilitating parasitic disease that represents a major health problem in endemic areas ${ }^{(39)}$. Its infection rates continue to be high in endemic regions and the overall prevalence either remains unchanged or has increased despite the global efforts to eradicate it by chemotherapy using $\mathrm{PZQ}^{(5)}$. So, the effective control of this parasite is unlikely to occur in the absence of improved sanitation and a vaccine ${ }^{(40)}$.

Schistosomes do not multiply within their mammalian hosts ${ }^{(10)}$. The targets of vaccination can be either the prevention of schistosome infection or the reduction of parasite number and fecundity. The gold standard for anti-schistosome vaccine development is the reduction in numbers of adult worms ${ }^{(8)}$.

In our study, we used a SWAP preparation that is used by immunologists to probe host responses to schistosome infections $^{(13)}$. It has been reported to induce antibodies capable of killing the schistosomulum in vitro ${ }^{(41)}$, and to convey partial protection in vivo ${ }^{(42)}$. Also, it was used to investigate human anti-fecundity immunity to $S$. haematobium using antibody isotype ELISAs as the assay of reactivity ${ }^{(43)}$. It is well established that the incorporation of an immunological adjuvant into a vaccine formulation, accelerates, prolongs or enhances the quality of specific immune responses to vaccine antigens ${ }^{(44)}$. So, the selection of a suitable adjuvant is a critical step in the development and employment of successful anti-schistosome vaccines ${ }^{(16)}$. The most commonly used adjuvant in the preclinical evaluation of schistosome vaccine candidates is $\mathrm{FCA}^{(40)}$; although it is unsuitable for use in human due to its side effects $^{(17,18)}$.

In a trial to explore a potential helper for the crude SWAP anti-schistosomal vaccine, we tested CSNPs as an adjuvant. We found that modified SWAP loaded on CSNPs, either alone or combined, had a greater impact against challenge of $S$. mansoni (groups 4 and 5), more than when using FCA with crude SWAP (group 3). Similar results were obtained by Etewa et al., ${ }^{(45)}$ who used mesenchymal stem cells as an adjuvant for a potential anti-schistosomal vaccine in murine models.

Recently, nanostructures are widely used in biomedical sciences because of their ability to cross the cell and tissue barriers due to their very small particle size ${ }^{(46)}$. They have significant advantages over the conventional drug delivery system regarding high stability, high specificity, high drug carrying capacity, possibility to use in different routes of administration, ability for controlled release and delivery of both hydrophilic and hydrophobic drug molecules ${ }^{(47)}$. Chitosan is soluble in diverse acids and able to interact with polyanions to form complexes and gels. It has anti- bacterial and anti-fungal properties and is safe and non-toxic ${ }^{(48)}$. CSNPs have been used as carriers of antitoxoplasmosis drugs ${ }^{(26)}$. Moreover, CSNPs produced $47 \%$ protection against $S$. mansoni infection in mice, suggesting its important role in inducing a protective immune response against schistosomiasis ${ }^{(49)}$. So, in the present study, we tested CSNPs as carriers (adjuvant) of a potential anti-schistosomal vaccine experimented in mice.

In the present study, efficacy of the crude SWAP antigen loaded on CSNPs was assessed parasitologically by estimation of stools egg count (modified Kato technique), worm burden (porto-mesenteric perfusion and ultrastructural studies) of both juvenile and adult stages. The results showed significant reduction in the number of $S$. mansoni eggs in stool samples 7 weeks PI in all tested subgroups in comparison to the control infected subgroup 2: The highest percentage reduction $(91.3 \%)$ was encountered with the combined antigens (crude SWAP+FCA+modified SWAP on CSNPs then infected; subgroup 5'). The current results agreed with Etewa et al., ${ }^{[15]}$ who found a significant reduction in the mean egg counts/g stools by Kato technique in combined antigens vaccinated group (crude soluble egg antigen (SEA) + crude SWAP), as compared to control infected group with percentage reduction of $90.3 \%$. On the other hand, Teixieira et al., ${ }^{(43)}$ reported reduction of $59-60 \%$ egg elimination in feces of mice immunized by schistosomula tegument (Smteg) supplemented with FCA.

The present work showed high significant reduction in the total worm burden in all tested groups compared with the infected control group. The maximum reduction in the juvenile and adult worms was recorded in the combined antigens subgroups (5 and 5) with percentage reduction of $64.3 \%$ and $85.4 \%$, respectively; but nearly the same efficacy of the combined vaccine was obtained by using SWAP loaded on CSNPs (novel preparation) which indicated its unique potency whether combined or alone (Tables 3 and 5). Also, Ismail ${ }^{(50)}$ reported $88 \%$ reduction in adult worm burden in combined antigens vaccinated mice. These findings are explained by Etewa et al., ${ }^{(15)}$ who stated that combining different antigens provides augmentation of the protective immunity compared to each component administered individually. On the other hand, a lower percentage in the reduction of adult worms $(40.3 \%)$ was detected by Ewaisha et al., ${ }^{(51)}$ on using a multi-antigen vaccine composed of recombinant $S m 14$ and a truncated form of $S m 29$, designated $\operatorname{Tr} S m 29$, in the presence of the immunoadjuvant poly (I:C). Accordingly, the researchers concluded that using multi-antigen vaccines is a more effective approach to fulfill enhanced protection against schistosomiasis than giving each component individually. Also, Teixieira et $a l .{ }^{(43)}$ reported a reduction in adult worm burden 
(43-48\%). These lower percentages may be due to the difference in the antigen preparation, adjuvants used or the Schistosoma strain.

Several studies were done to develop new schistosomicides to overcome the emergence of PZQ resistance. Abdel-Ghaffar et al., ${ }^{(52)}$ showed a significant reduction in both schistosomula and adult stage worm burden with maximum reduction in mice treated with artemisinin combined with PZQ with percentage reduction of $60.4 \%$ and $92.7 \%$, respectively. Also, a $93.7 \%$ reduction in adult worm burden was detected in mice treated with Nigella sativa combined with $\mathrm{PZQ}^{(53)}$. These results indicate the vulnerability of adult worms to various anti-schistosomal preparations. Our current work resulted in significant reduction of couples with maximum reduction in the combined antigens subgroup (5') (R 93.3\%). The results obtained by Abououf et al., ${ }^{(53)}$ showed that N. sativa combined with PZQ had a highly significant reduction rate of coupled worms (R 91.7\%). These findings emphasize indirectly our results and potentiate the immune impact of the tested antigens that may lead to couple separation.

In our present study, male worms were more affected than females with maximum reduction in the combined antigens subgroup (R 89\% and 81.5\%, respectively) (Table 5). Also, Ali et al., ${ }^{(54)}$ on using $N$. sativa and Chroococcus turgidus extract to evaluate their effects against $S$. mansoni, they reported higher decrease in male worm burden than female one. However, El Bialy et al., ${ }^{(55)}$ assessed a novel benzimidazole-derived compound against $S$. mansoni, and reported significant reductions in the number of adult worms especially females. El-Lakkany et al., ${ }^{(56)}$ attributed such variation of males and females' numbers, to differences in $S$. mansoni strain used in each study. In our opinion, the cercarial infective dose should also be considered.

The tegument of schistosomes is a living, anucleate, and cytoplasmic structure, attached to underlying cell bodies (cytons) by narrow cytoplasmic connections. These cytons synthesize the discoid bodies and multilaminate vesicles. The function of these vesicles is to build up and maintain the continuously shed tegument ${ }^{(57)}$. It is involved in nutrient absorption, presents proteins that are responsible for evasion of the host immune response ${ }^{(58)}$, and it is the interface between the parasite and the host environment. So, the tegument is an important target for drugs and vaccines action. Currently, we studied the effect of immunization with crude SWAP plus different adjuvants preparations, on the tegument of juvenile and adult $S$. mansoni worms by SEM, which gave clear morphological examination with direct visualization and offered several advantages in morphological and sizing analysis ${ }^{(47)}$. Our SEM study of the effect of vaccinations on the schistosomula of $S$. mansoni revealed a variable degrees of tegumental change; tegumental tubercles were less developed and irregularly distributed; some tubercles were without spines; others had stunted apically directed spines; there were detached parts of the tegument, and the inter-tubercular ridges were less prominent. The damaged tegumental surface was collapsed. There were areas of tegumental erosion with irregular edges of gynecophoric canal. The suckers showed blebs and swelling. Red blood cells and irregular big cells were seen adherent to the tegument. The most severe tegumental changes were in the combined antigens subgroup (5`).

Our results are in parallel with Abdel-Ghaffar et al., ${ }^{(52)}$ who due to the use of anti-schistosomal therapeutic preparations, reported marked SEM schistosomula tegumental changes. Changes were in the form of numerous blebs protruding from the surface of the tegument with moderate to severe swelling, peeling, and erosion of the tegument. Also, Fakahany et al.,(59) observed tegumental damage following mefloquine treatment.

In the present work, there was a significant distortion in the tegument of male and female worms in different tested subgroups compared to infected control subgroup. The subgroups 4 and 5 ' that were immunized by SWAP whether loaded on CSNPs or combined with crude SWAP and FCA showed marked extensive tegumental alterations on recovered adult worms. These alterations presented as erosion, disruption of tubercles, swelling, blebbing, pitting and sometimes detachment of the tegument with partial and mostly loss of the tubercular spines (Figs. 12-19). Tubercle distortions causes inability of worm adherence to host's walls of blood vessels, that lead to dislodgement and transportation of worms in the blood stream from mesenteric veins to portal vein and intravenous hepatic capillaries and lodgment in the liver ${ }^{(60)}$. Anatomical and morphological changes in gynecophoric canal that may result in separation of males and females were shown in figures 12 and 15. The female tegument in figure (17) showed banded appearance because of the loss of normal ridged topography, and the loss of tubercles in vast localities left tegumental surface pits. The tegumental affection is a good sign of the potency of the used antigen preparations which may expose the subtegumental layer and disturb the physiologic functions of the tegument.

Adult worms in the combined antigens subgroup 5 ' were more contracted and shortened in comparison to subgroup 4' (Fig. 15), the worms showed kinking and bending (Fig. 18); this is caused by the severe tegumental damage and the extensive erosion with adherence of the host immune cells (Fig. 17). Contact with such an immunologically unprotected surface, leads to abnormal structural changes with disturbance of calcium $\left(\mathrm{Ca}^{2+}\right)$ channels. The adult worms undergo 
an increased $\mathrm{Ca}^{2+}$ influx, followed by intense and sustained muscular contraction, disruption of the parasite physiological functions and death ${ }^{(61)}$.

The results of our work were closely related to Abdel-Zaher et al., ${ }^{(14)}$ who reported that the severest ultrastructural changes in adult $S$. mansoni tegument were in mice immunized with polyvalent vaccine (CAP, SWAP, and SEA). While, Abououf et al., ${ }^{(53)}$ reported that $N$. sativa oil alone or combined with PZQ showed structural changes in tegument of the adult treated worms. Similar results were obtained by El-Shafey et al., ${ }^{(62)}$ on using different doses of artemether (ART) to assess its effects on the tegument of adult $S$. mansoni worms in vitro. They observed marked tegumental changes after exposure to $100 \mu \mathrm{g} / \mathrm{ml}$ ART. These findings substantiate that tegumental damage is the intended goal of both anti-schistosomal therapeutics and vaccination.

Our results of immunization with SWAP, crude or loaded on CSNPs or combined, induced distortion in the tegument of adult worms, disruption with dilatation and expansion of the borders of gynecophoric canals which may lead to separation of the female worms. That is besides the adverse effects on female maturity and fecundity. These recorded changes correspond to previous SEM observations obtained by Xiao et al., ${ }^{(58)}$.

In our study, the abnormal morphological changes were first observed in the tegument of male worms, which is attributed to the more pronounced soft tissue alterations in males than in females ${ }^{(63)}$. Therefore, the tegumental changes were apparently more prominent in male worms. Similar results were obtained by AbdelZaher et al., ${ }^{(14)}$ and de Moraes et al., ${ }^{(64)}$. Also, El-Beshbishi et al., ${ }^{(65)}$, in their in vitro study on $S$. haematobium adult worms using Artemisinin-naphthoquine phosphate (CO-ArNp) as therapy, they found extensive tegumental alterations mainly in treated male schistosomes. On the other hand, El Bialy et al., ${ }^{(55)}$ and Fakahany et al., ${ }^{(59)}$ reported that the tegument of female worms was more affected compared to male worms.

In conclusion, the present study has shown that immunization by combining different antigens [crude SWAP + FCA and modified SWAP on CSNPs] gave the best results in terms of reduction of worm load and fecundity with prominent tegumental alterations, than when each component was administered individually. However, more work is needed to confirm our results and further assessment is still needed in these aspects.

Acknowledgments: The authors would like to express their gratitude to the Electron Microscope Unit, Faculty of Agriculture, Mansoura University personnel, who helped in preparation, examination and photography of the ultrastructural study.
Author Contribution: SE Etewa, AA Al-Hoot and HM Sharaf conceived and designed the presented idea. SM Mohammad, HSF Moawad, MH Sarhan, MA El-Shafey and HS Senousy were responsible for acquisition, analysis and interpretation of resulting data. SM Mohammad, HSF Moawad and MH Sarhan wrote the draft of the manuscript. AA Al-Hoot and MH Sarhan completed the critical revision of the article. SE Etewa approved the final version for publication.

Conflict of interest: There is no conflict of interest.

Financial support and sponsorship: Nil.

\section{REFERENCES}

1. El Ridi R, Tallima H. Schistosoma mansoni ex vivo lung-stage larvae excretory-secretory antigens as vaccine candidates against schistosomiasis. Vaccine 2009; 27(5): 666-673.

2. King $\mathrm{CH}$, Dickman K, Tisch DJ. Reassessment of the cost of chronic helminthic infection: a metaanalysis of disability-related outcomes in endemic schistosomiasis. Lancet 2005; 365(9470): 15611569.

3. Steinmann P, Keiser J, Bos R, Tanner M, Utzinger J. Schistosomiasis and water resources development: systematic review, meta-analysis, and estimates of people at risk. Lancet Infect Dis 2006; 6(7): 411425.

4. Schur N, Hürlimann E, Garba A, Traoré MS, Ndir 0 , Ratard RC, et al. Geostatistical model-based estimates of schistosomiasis prevalence among individuals aged $\leq 20$ years in West Africa. PLoS Negl Trop Dis 2011; 5(6): e1194.

5. Gray DJ, McManus DP, Li YS, Williams GM, Bergquist $\mathrm{R}$, Ross AG. Schistosomiasis elimination: lessons from the past guide the future. Lancet Infect Dis 2010; 10: 733-736.

6. N'Goran EK, Utzinger J, Gnaka HN, Yapi A, N'Guessan NA, Kigbafori SD, et al. Randomized, double-blind, placebo-controlled trial of oral artemether for the prevention of patent Schistosoma haematobium infections. Am J Trop Med Hyg 2003; 68(1): 24-32.

7. Rollinson D, Knopp S, Levitz S, Stothard JR, Tchuem Tchuenté LA, Garba A, et al. Time to set the agenda for schistosomiasis elimination. Acta Tropica 2013; 128(2): 423-440.

8. McManus DP, Loukas A. Current status of vaccines for schistosomiasis. Clin Microbiol Rev 2008; 21(1): 225-242. 
9. Zhang W, Ahmad G, Torben W, Siddiqui AA. Schistosoma mansoni antigen Sm-p80: Prophylactic efficacy of a vaccine formulated in human approved plasmid vector and adjuvant (VR 1020 and alum). Acta Tropica 2011; 118(2), 142-151.

10. Gryseels B, Polman K, Clerinx J, Kestens L. Human schistosomiasis. Lancet 2006; 36: 1106-1118.

11. Chitsulo L, Loverde P, Engels D. Schistosomiasis. Nat Rev Microbiol 2004; 2(1): 12-13.

12. Van Lieshout L, Polderman AM, Deelder AM. Immunodiagnosis of schistosomiasis by determination of the circulating antigens CAA and CCA, in particular in individuals with recent or light infections. Acta Tropica 2000; 77(1): 69-80.

13. Neves LX, Sanson AL, Wilson RA, Castro-Borges W. What's in SWAP? Abundance of the principal constituents in a soluble extract of Schistosoma mansoni revealed by shotgun proteomics. Parasit Vectors 2015; 8:337.

14. Abdel-Zaher M, Abed GH, Abdel-Hakeem SS. Ultrastructural changes of Schistosoma mansoni worms associated with administration of its polyvalent vaccine. J Zool Stud 2016; 3(6): 09-20.

15. Etewa SE, Hegab MH, Metwally AS, Abd Allah SH, Shalaby SM, El-Shal AS, et al. A molecular approach for evaluation of experimental trials of anti schistosomal vaccination in murine models. AfroEgypt J Infect Endem Dis 2016; 6(3): 142-151.

16. Khalifa RMA, Elnadi NA, Omran EK, Abdel-Tawab RA. Immunological response and the probability of production of vaccine for schistosome parasites. Egypt J Med Sci 2011; 32(2): 547-570.

17. Handman E. Leishmaniasis: current status of vaccine development. Clin Microbiol Rev 2001; 14(2): 229-243.

18. Hacariz O, Sayers G, McCullough M, Garrett M, O’Donovan J, Mulcahy G. The effect of Quil adjuvant on the course of experimental Fasciola hepatica infection in sheep. Vaccine 2009; 27: 45-50.

19. Gregory AE, Titball R, Williamson D. Vaccine delivery using nanoparticles. Front Cell Infect Microbiol 2013; 3: 1-13.

20. Bhatia S. Nanoparticles types, classification, characterization, fabrication methods and drug delivery applications. In: Bhatia S (Ed.) Natural polymer drug delivery systems. Springer International Publishing, Switzerland, 2016; pp 33-93.
21. Ing LY, Zin NM, Sarwar A, Katas H. Antifungal activity of chitosan nanoparticles and correlation with their physical properties. Int J Biomater 2012; 2012:632698.

22. Danesh-Bahreini MA, Shokri J, Samiei A, KamaliSarvestani E, Barzegar-Jalali M, MohammadiSamani S. Nanovaccine for leishmaniasis: preparation of chitosan nanoparticles containing Leishmania superoxide dismutase and evaluation of its immunogenicity in BALB/c mice. Int J Nanomedicine 2011; 6: 835-842.

23. Kean T, Thanou M. Biodegradation, biodistribution and toxicity of chitosan. Adv Drug Deliv Rev 2010; 62(1): 3-11.

24. Said DE, Elsamad LM, Gohar YM. Validity of silver, chitosan, and curcumin nanoparticles as antiGiardia agents. Parasitol Res 2012; 111(2): 545554.

25. Ali M, Afzal M, Verma M, Misra-Bhattacharya S, Ahmad FJ, Dinda AK. Improved anti-filarial activity of ivermectin in chitosan-alginate nanoparticles against human lymphatic filarial parasite, Brugia malayi. Parasitol Res 2013; 112(8): 2933-2943.

26. Etewa SE, Abo El-Maaty DA, Hamza RS, Metwaly AS, Sarhan MH, Abdel-Rahman SA, et al. Assessment of spiramycin-loaded chitosan nanoparticles treatment on acute and chronic toxoplasmosis in mice. J Parasit Dis 2018; 42(1): 102-113.

27. El-Gawish MA, Hafez MN, Eid FA, Soliman MG, Khalil SM. Efficiency of immunization of mice with irradiated antigen against Schistosoma mansoni infection in comparison with praziquantel. EJHM 2006; 25: $630-655$.

28. Salih SY, Bartlett A, Voller A. Detection of antibodies by enzyme-immunoassay in human Schistosoma mansoni infections: a clinical and chemotherapeutic study. Tropenmed Parasitol 1978; 29(4): 409-412.

29. Jarudilokkul S, Tongthammachat A, Boonamnuayvittaya V. Preparation of chitosan nanoparticles for encapsulation and release of protein. Korean J Chem Eng 2011; 28(5): 1247-1251.

30. Liang YS, John I, Bruce JI, David AB. Laboratory cultivation of schistosome vector snails and maintenance of schistosome life cycle. Proc First Sine Am Symp 1987; 1: 34.

31. Webbe G, James G. The importation and maintenance of schistosomes of human and veterinary importance. In: Taylor AER and Muller R Eds. Isolation and maintenance of parasites in vivo. 
$9^{\text {th }}$ symposium of British Society of Parasitology. Oxford-Blackwell Scientific Publications, London, $1971 ; 77-107$.

32. Elkhafif N, Voss B, Hammam O, Yehia H, Mansy S, Akl $\mathrm{M}$, et al. Homing of transplanted bone marrow cells in livers of Schistosoma mansoni-infected mice. APMIS 2010; 118(4):277-287.

33. Nabih I, Soliman AM. Studies on fresh water snails, specific intermediate host for schistosomiasis. II. Isolation of total protein from native and irradiated snails. Cell Mol Biol 1986; 32: 315- 317.

34. Smithers SR, Hackett F, Ali OP, Simpson AJG. Protective immunization of mice against Schistosoma mansoni with purified adult worm surface membranes. Parasite Immunol 1989; 11: 301-318.

35. Martin LK, Beaver PC. Evaluation of kato thicksmear technique for quantitative diagnosis of helminth infection. Am J Trop Med Hyg 1968; 17: 382-391.

36. Smithers SR, Terry RJ. The infection of laboratory hosts with cercariae of Schistosoma mansoni and the recovery of the adult worms. Parasitology 1965; 55(4): 695-700.

37. Fallon PG, Sturrock RF, Niang AC, Doenhoff MJ. Short report: diminished susceptibility to praziquantel in a Senegal isolate of Schistosoma mansoni. Am J Trop Med Hyg 1995; 53(1): 61-62.

38. Machado-Silva JR, Galvão C, Oliveira RMF, Presgrave OAF, Gomes DC. Schistosoma mansoni Sambon, 1907: comparative morphological studies of some Brazilian strains. Rev Inst Med Trop Sao Paulo 1995; 37:441-443.

39. Schneider MC, Aguilera XP, Barbosa da Silva Junior J, Ault SK, Najera P, Martinez J, et al. Elimination of neglected diseases in Latin America and the Caribbean: A Mapping of Selected Diseases. PLoS Negl Trop Dis 2011; 5(2): e964.

40. Tran MH, Pearson MS, Bethony JM, Smyth DJ, Jones MK, Duke M, et al. Tetraspanins on the surface of Schistosoma mansoni are protective antigens against schistosomiasis. Nat Med 2006; 12: 835840.

41. Sher A, Kusel JR, Perez H, Clegg JA. Partial isolation of a membrane antigen which induces the formation of antibodies lethal to schistosomes cultured in vitro. Clin Exp Immunol 1974; 18(3): 357-369.

42. Teixeira de Melo T, Michel de Araujo J, Do Valle Durães F, Caliari MV, Oliveira SC, Coelho PM, et al. Immunization with newly transformed Schistosoma mansoni schistosomula tegument elicits tegument damage, reduction in egg and parasite burden. Parasite Immunol 2010; 32(1112): 749-759.

43. Wilson S, Jones FM, van Dam GJ, Corstjens PL, Riveau G, Fitzsimmons CM, et al. Human Schistosoma haematobium anti-fecundity immunity is dependent on transmission intensity and associated with immunoglobulin G1 to worm-derived antigens. J Infect Dis 2014; 210(12): 2009-2016.

44. Vogel FR. Improving vaccine performance with adjuvants. Clin Infect Dis 2000; 30: 266-270.

45. Etewa SE, Abd Allah SH, Badawey MS, Shalaby SM, El-Shal AS, El Shafey MA, et al. The effect of stem cells as an adjuvant on the immunogenicity of a potential anti-schistosomal vaccine in mice. J Egypt Soc Parasitol 2016; 46(3): 693-716.

46. Dou Z, Wang G, Zhang E, Ning F, Zhu Q, Jiang J, et al. Effect of $\mathrm{Al} 2 \mathrm{O} 3$ nanoparticles doping on the microwave dielectric properties of CTLA ceramics. J Material Sci Eng 2016; 5:256.

47. Pal SL, Jana U, Manna PK, Mohanta GP, Manavalan R. Nanoparticle: An overview of preparation and characterization (2000-2010). J Appl Pharm Sci 2011; 1(06): 228-234.

48. Kim S-K, Rajapakse N. Enzymatic production and biological activities of chitosan oligosaccharides (COS): A review. Carbohydrate Polymers 2005; 62(4): 357-368.

49. Oliveira CR, Rezende CMF, Silva MR, Borges OM, Pego AP, Goes AM. Oral vaccination based on DNA-chitosan nanoparticles against Schistosoma mansoni infection. Sci World J 2012; 11: 938457.

50. Ismail OA. Study of the efficacy of adult worm, cercarial and egg antigens in protection against experimental intestinal schistosomiasis. MD Thesis, Medical Parasitology Department, Faculty of Medicine, Suez Canal University, Egypt, 2005.

51. Ewaisha RE, Bahey-El-Din M, Mossallam SF, Amer EI, Aboushleib HM, Khalil AM. Combination of the two schistosomal antigens $\operatorname{Sm} 14$ and Sm29 elicits significant protection against experimental Schistosoma mansoni infection. Exp Parasitol 2014; 145: 51-60.

52. Abdel-Ghaffar MM, Saad AE, Moharm IM, Sharaf OF, Badr MT, Ibrahim AF. Parasitological and histopathological effects of some anti-schistosome 
drugs in Schistosoma mansoni-infected mice. MM] 2018; 30: 1193-1202.

53. Abououf EA, Elhamshary AMS, Nagati IM, Eraky MA, Elkholy AA, Ibrahim AN, et al. Effect of Nigella sativa oil on Schistosoma mansoni mature worms in experimentally infected mice. J Egypt Soc Parasitol 2018; 48(1): $55-66$.

54. Ali M, Eldahab MA, Mansour HA, Nigm A. Schistosoma mansoni: Antiparasitic effects of orally administered Nigella sativa oil and/or Chroococcus turgidus extract. Acta Biol Hung 2016; 67(3): 247260.

55. El Bialy SA, Taman A, El-Beshbishi SN, Mansour B, El-Malky M, Bayoumi WA, et al. Effect of a novel benzimidazole derivative in experimental Schistosoma mansoni infection. Parasitol Res 2013; 112(12): 4221-4229.

56. El-Lakkany NM, El-Din SH, Sabra AN, Hammam OA. Pharmacodynamics of mefloquine and praziquantel combination therapy in mice harboring juvenile and adult Schistosoma mansoni. Mem Inst Oswaldo Cruz 2011; 106(7): 814-822.

57. Van Hellemond JJ, Retra K, Brouwers JF, van Balkom BW, Yazdanbakhsh M, Shoemaker CB, et al. Functions of the tegument of schistosomes: clues from the proteome and lipidome. Int J Parasitol 2006; 36(6): 691-699.

58. Xiao SH, Shen B, Utzinger J, Chollet J, Tanner M. Ultrastructural alterations in adult Schistosoma mansoni caused by artemether. Mem Inst Oswaldo Cruz 2002; 97:717-724.

59. Fakahany AF, Younis MS, El Hamshary AMS, Fouad MAH, Hassan MAE, Ali HSM. Effect of mefloquine on worm burden and tegumental changes in experimental Schistosoma mansoni infection. J Microsc Ultrastruct 2014; 2(1): 7-11.

60. Mehlhorn H, Becker B, Andrews $\mathrm{P}$, Thomas $\mathrm{H}$, Frenkel JK. In vivo and in vitro experiments on the effects of praziquantel on Schistosoma mansoni: a light and electron microscopic study. Arzneimittelforschung 1981; 31(3a): 544-554.

61. Pax R, Bennett JL, Fetterer R. A benzodiazepine derivative and praziquantel: effects on musculature of Schistosoma mansoni and Schistosoma japonicum. Naunyn Schmiedebergs Arch Pharmacol 1978; 304(3): 309-315.

62. El Shafey MA, Etewa SE, Sarhan MH, Arafa SZA, Abo El-Maaty DA, Al-Hoot AAA. Ultrastructural and muscular modifications of adult Schistosoma mansoni worms induced by artemether (ART) in vitro. J Egypt Soc Parasitol 2018; 48(1):101-107.

63. Mostafa OMS, Soliman MI. Ultrastructure alterations of adult male Schistosoma mansoni harbored in albino mice treated with Sidr honey and/or Nigella sativa oil. J King Saud Univ Sci 2010; 22(3): 111-121.

64. de Moraes J, Nascimento C, Miura LM, Leite JR, Nakano E, Kawano T. Evaluation of the in vitro activity of dermaseptin 01 , a cationic antimicrobial peptide, against Schistosoma mansoni. Chem Biodivers 2011; 8(3): 548-558.

65. El-Beshbishi SN, El Bardicy S, Tadros M, Ayoub M, Taman A. Efficacy of artemisinin-naphthoquine phosphate against Schistosoma haematobium adult flukes: dose-effect relationship and tegumental alterations. J Helminthol 2018; 21: 1-6 